\section{Design-based methodological advances to support national forest inventories: a review of recent proposals}

\section{Lorenzo Fattorini}

The aim of this paper is to give an overview of some recent proposals to support national forest inventories. The reviewed literature is strictly of designbased nature, i.e., uncertainty only stems from the sampling scheme actually adopted in the survey, rather than being assumed or modeled as in modelbased approaches. National forest inventories are viewed as two-phase sample surveys to estimate at the same occasion the extent of the continuous population of points constituting the forest cover and the total of a forest attribute (e.g., volume or biomass) in the discrete population of trees for several forest types and/or administrative districts. The first phase is performed from remote sensing imagery while the second phase is performed on the field, possibly adopting the information acquired in the first phase as auxiliary information. A novel methodology is adopted based on Monte Carlo integration methods, which leads to a very general estimation strategy. Some recent proposals are considered in which remote sensing information acquired in the first phase is used to assess some physical characteristics of non-forest resources, such as woodlots, tree-rows and isolated trees outside the forest without additional field work. Finally, a new proposal is discussed in which canopy height from laser scanning is adopted as auxiliary information to recover missing data occurring when some sampled points cannot be reached because of hazardous terrain.

Keywords: Two-phase Strategies, Aerial Information, Non-forest Resources, Missing Data, LiDAR, Calibration Weighting

\section{Introduction}

The analysis of forest ecology and the wise management of forest resources requires accurate monitoring of forestlands at regular time intervals. In turn, the monitoring process involves sample surveys to estimate extents and standing volumes for a wide set of forest types and districts. These surveys are usually referred to as forest inventories (Corona \& Marchetti 2007, Corona et al. 2011a).

The early forest inventories were performed toward the end of $15^{\text {th }}$ century in the

$\square$ Department of Economics and Statistics, University of Siena, p.za S. Francesco 8, I-53100 Siena (Italy)

@ Lorenzo Fattorini

(lorenzo.fattorini@unisi.it)

Received: Jan 08, 2014 - Accepted: Mar 11, 2014

Citation: Fattorini L, 2015. Design-based methodological advances to support national forest inventories: a review of recent proposals. iForest 8: 6-11 [online 2014-0618] URL: http://www.sisef.it/iforest/ contents/?id=ifor1239-007

Communicated by: Marco Borghetti phase sample surveys to estimate at the same occasion the extent of the continuous population of points constituting the forest cover and the total of a forest attribute (e.g., volume or biomass) in the discrete population of trees for several forest types and/or administrative districts. Usually, NFIs are performed using an intensive first phase which is carried out on satellite imagery or aerial photos and a second phase which is carried out by ground inspections, possibly adopting the information acquired in the first phase as auxiliary information. A novel methodology is adopted based on Monte Carlo integration methods (Gregoire \& Valentine 2008, Mandallaz 2008), which leads to a very general estimation strategy valid for any second-phase sampling scheme. In section 3, some recent proposal are considered in which the aerial information acquired in the first NFI phase is exploited to investigate non forest resources, such as woodlots, tree-rows and isolated trees outside the forest. In section 4 a new proposal is discussed in which canopy height from laser scanning is adopted as auxiliary information to account for missing data occurring when some sampled points cannot be reached by forest crews because of hazardous terrain. Final remarks are given in section 5 .

\section{Two-phase inventories}

Consider a delineated study area A partitioned into two land cover classes: forest and non forest. Denote by $\mathrm{F} \subset \mathrm{A}$ the forest portion of $\mathrm{A}$ and by $\mathrm{U}$ the population of forest trees within $F$. Suppose that $F$ is partitioned into $K$ sub-portions $\mathrm{F}_{l}, \ldots, \mathrm{F}_{K}$ corresponding to $K$ forest types (e.g., oak, pine, larch, etc.) or $K$ spatial districts or combinations of the twos, in such a way that $U$ is partitioned into $K$ sub-populations $\mathrm{U}_{l}, \ldots, \mathrm{U}_{K}$ of trees within the $K$ sub-portions.

Generally speaking, a forest inventory is a sampling strategy to estimate the extent of the $k$-th forest type/district (eqn. 1):

$$
A_{k}=\int_{\mathrm{A}} y_{k}(p) d p
$$

perormed in Finland from 1922 to 1924 and from now repeated every ten years. The first Italian NFI was performed from 1982 to 1985, while the second started in 2003 and concluded at the end of 2006 (see http:// www.infc.it).

This paper provides a review of some recent methodological contributions to support NFIs. All the reviewed articles are of designbased nature, in the sense that uncertainty only stems from the sampling scheme adopted to perform the inventory. As Särndal et al. (1992) point out "Design-based inference is objective; nobody can challenge that the sample was really selected according to the given sampling design. The probability distribution associated with the design is real, not modelled or assumed".

In the next section NFIs are viewed as two- and the total of a forest attribute $Y$ (e.g., volume, biomass, basal area, etc. - eqn. 2):

$$
T_{k}=\sum_{j \in \mathrm{U}_{k}} y_{j}
$$

for each $k=1, \ldots, K$, where $y_{k}(p)$ is the indicator variable of the $k$-th forest type/category defined in the continuous population of points $p \in \mathrm{A}$ and is such that $y_{k}(p)=1$ if $p \in$ A and $y_{k}(p)=0$ otherwise, while $y_{j}$ is the amount of $Y$ corresponding to the $j$-th tree in the discrete population of trees $U$.

An essential requirement of any NFI is that extents and totals are estimated in the same survey, using the same aerial and field investigations. 


\section{First phase}

Gregoire \& Valentine (2008) and Mandallaz (2008) provide excellent introductory treatments on the issue of sampling discrete objects (forest trees in the present case) scattered over a region by means of plots or transects (plots in the present case) to estimate a total. The authors emphasize that these sampling strategies may be conveniently re-formulated as spatial strategies for sampling the continuous populations of points constituting the study area and estimating an integral within. Gregoire \& Valentine (2008) also provide a list of references from the early 1990s in which such a novel intuition was first developed. In this setting, the inventory issue of estimating both extents and totals is unified, because just like extents, the totals can be expressed as integrals over the study area. The difference between extents and totals is due to the variable to be recorded. As to extents, the survey variable is the indicator variable at the sampled point; as to totals, the survey variable is the whole amount of the forest attribute $Y$ recorded for the trees lying within the plot of prefixed size and shape centred at the sampled point. Accordingly, in the first phase a unique spatial design for selecting points (from which plots are centred) suffices for both extent and total estimation. As pointed out by Gregoire \& Valentine (2008) and Mandallaz (2008), in this framework estimation reduces to a twodimensional Monte Carlo integration. Thus the key problem is how to effectively select the first-phase points to better perform integration.

Despite its simplicity, the completely random placement of $N$ first-phase points, usually referred to as the uniform random sampling (URS), may lead to uneven coverage of the study area, because some parts of the area may be sparsely sampled whereas others are intensively sampled. To avoid the drawback, stratified or systematic schemes can be adopted. A stratified scheme, usually referred to as the tessellation stratified sampling (TSS) is performed as follows: the area A is covered by a region $\mathrm{R} \supset \mathrm{A}$ of size $R$, constituted by $N$ non-overlapping regular polygons of equal size $\mathrm{R}_{l}, \ldots, \mathrm{R}_{N}$, and such that $\mathrm{R}_{i} \cap \mathrm{A} \neq \varnothing$ for all $i=1, \ldots, N$. Then, for each polygon $i$, a point is randomly thrown within the polygon. Alternatively, a systematic scheme, usually referred to as the systematic grid sampling (SGS) can be used: in this case a point is randomly selected in one polygon (e.g., the first) and then repeated in the remaining $N-1$. Most of the NFIs adopted the SGS scheme, while TSS has been recently applied in the last Italian NFI (Fattorini et al. 2006)

If each first-phase point is visited on the ground and the indicator variable of each type/district is recorded at the point, the first-phase Monte-Carlo integration estima- tor of $\mathrm{A}_{k}$ turns out to be (eqn. 3):

$$
\hat{A}_{(1) k}=\frac{R}{a} f_{k}
$$

where $f_{k}=N_{k} / N$ and $N_{k}$ is the number of sample points lying in $\mathrm{A}_{k}$. Moreover, if for each first-phase point $i$ lying in $\mathrm{A}_{k}$ a plot of fixed size $a$ is delineated around the point, the attribute $Y$ is recorded for all the trees in the plots, and the total $t_{k i}$ is reckoned, the first-phase Monte-Carlo integration estimator of $T_{k}$ turns out to be (eqn. 4):

$$
\hat{T}_{(1) k}=\frac{R}{a} \frac{1}{N} \sum_{i=1}^{N} t_{k i}
$$

where $t_{k i}=0$ if $i$ does not belong to $\mathrm{A}_{k}$.

It is well-known from Monte Carlo integration (Gregoire \& Valentine 2008) that (3) and (4) are unbiased estimators under URS, STS and SGS schemes. Moreover, under TSS and SGS, the variances of (3) and (4) decrease at a rate faster than $N^{-1}$ (Barabesi 2003, Barabesi \& Marcheselli 2003, Barabesi \& Franceschi 2011), while URS provides variances decreasing with $N^{-1}$. Accordingly, for large $N$, tessellation gives rise to relevant gains in precision with respect to URS.

It is worth noting that some edge effects may be present owing to forest trees positioned near the edge of the study region, which have inclusion probabilities smaller than the inner trees. A long list of correction methods has been proposed in order to avoid the negative bias induced by edge effects (Gregoire \& Valentine 2008). Fortunately, in this framework, the TSS and SGS schemes, selecting first-phase points onto the enlarged region $\mathrm{R}$, perform like the correction method usually referred to as the buffer method (Gregoire \& Valentine 2008), i.e., the $N$ points are allowed to fall outside the boundary of A, but within some larger region including A. For this reason, under TSS and SGS the presence of forest trees whose inclusion zone overlaps the boundary of the enlarged region $\mathrm{R}$ is likely to be negligible. Moreover, it should be noticed that in NFIs edges coincide with the country's borderlines, i.e., mountains ridges, rivers, sea in which the presence of forest trees is very unlike to occur. Thus, if edge effects are ignored that should not entail detrimental effects on the bias of the estimators.

\section{Second phase}

Owing to costs and time involved, in real situations the $N$ points selected in the first phase cannot be visited. Rather, only a portion of these points is selected in a second phase of sampling and is visited on the ground. Actually, the first-phase is only hypothetical and its treatment has the sole aim to lead to the estimators arising from the second phase.

Regarding the second phase, the collection of the $N$ points selected in the first phase, say
$\mathrm{P}$, is usually partitioned into the sub-set $\mathrm{P}_{F}$ of the $N_{F}$ points lying in the forest area $\mathrm{F}$, and the sub-set $\mathrm{P}-\mathrm{P}_{F}$ of the remaining $N-N_{F}$ points lying outside. It is worth noting that the partition is performed by satellite imagery of aerial photos, without field work. Obviously, because the plots centered at the points of P- $\mathrm{P}_{F}$ lie completely or partially outside forest, no or very few forest trees are likely to be found in these plots. Hence, it is customary to assume $t_{k i}=0$ for any $i \in \mathrm{P}-\mathrm{P}_{F}$, in such a way that the sampling effort can be completely devoted to $\mathrm{P}_{F}$.

The procedure of neglecting non-forest points in the second phase is adopted in most NFIs but it is not sufficiently focused in most familiar textbooks (de Vries 1986, Schreuder et al. 1993, Gregoire \& Valentine 2008, Mandallaz 2008). Even if the procedure is suitable from both theoretical and practical point of view because avoids the waste of sampling effort outside forest, it may provide downward bias in both extent and total estimation. Indeed, a point can be erroneously classified outside forest by aerial imaging. The issue of aerial classification errors is well known in forest inventorying, and first-phase errors can be accounted for in the second phase, achieving unbiased estimators of extent (Fattorini et al. 2004). However, if points classified as non-forest are excluded from the second phase of sampling, there is no way to account for those forest points erroneously classified as non-forest. This fact obviously entail underestimation of forest extents. At least to my knowledge, there is no inquiry or case study attempting to quantify how huge is the downward bias due to misclassification. To reduce misclassification effects, the first phase should include more auxiliary data sets as possible (e.g., maps, point clouds from airborne laser scanning, forest management plans etc.). If the aerial imagery is a high spatial resolution photographs, the non-forest areas should be quite easily distinguished from forest. In the same way, a plot centered at a point falling outside forest but near a forest stand, may contain a portion of forest trees which cannot be accounted because the point is excluded from the second phase. This fact obviously entail underestimation of totals. Some simulation studies (Maffei 2011, Corona et al. 2014) have evidenced that the bias induced by the exclusion of non-forest points is negligible.

If non-forest points are discarded, denote by $\mathrm{S} \subset \mathrm{P}_{F}$ the second-phase sample of size $n$ selected from $\mathrm{P}_{F}$ by means of a fixed-size scheme inducing first- and second-order inclusion probabilities $\pi_{i}$ and $\pi_{i h}\left(h>i \in \mathrm{P}_{F}\right)$. Suppose that $\pi_{i h}>0$ for any $h>i \in \mathrm{P}_{F}$, in such a way that the second-phase variance can be unbiasedly estimated. Suppose also that no classification errors between forest and non-forest points occur, and that $\operatorname{Pr}\left(t_{k i}=\right.$ 
$\left.0 \forall i \in \mathrm{P}-\mathrm{P}_{F}\right)=1$. Using the double-expansion estimation (Särndal et al. 1992), the two-phase estimators of extents and totals turn out to be (eqn. 5):

$$
\hat{A}_{(2) k}=\frac{R}{a} \frac{1}{N} \sum_{i \in \mathrm{S}_{k}} \frac{1}{\pi_{i}}
$$

and (eqn. 6):

$$
\hat{T}_{(2) k}=\frac{R}{a} \frac{1}{N} \sum_{i \in \mathrm{S}_{k}} \frac{t_{k i}}{\pi_{i}}
$$

respectively, where $\mathrm{S}_{k} \subset \mathrm{S}$ denotes the subsample of second-phase points lying in the $k$ th forest type/district.

Under the above-mentioned assumptions, estimators (5) and (6) turn out to be unbiased with sampling variances which in the case of (5) can be estimated by (eqn. 7):

$$
\begin{gathered}
V_{(2) k}=\left(\frac{R}{a}\right)^{2} \frac{1}{N^{2}} . \\
\left(\sum_{i \in \mathrm{S}_{k}} \frac{1}{\pi_{i}^{2}}+2 \sum_{h>i \in \mathrm{S}_{k}} \frac{1}{\pi_{i} \pi_{h}}-2 \frac{N}{N-1} \sum_{h>i \in \mathrm{S}_{k}} \frac{1}{\pi_{i h}}\right)
\end{gathered}
$$

and in the case of (6) by (eqn.8):

$$
V_{(2) k}=\left(\frac{R}{a}\right)^{2} \frac{1}{N^{2}} \text {. }
$$

$\cdot\left(\sum_{i \in \mathrm{S}_{k}} \frac{t_{k i}^{2}}{\pi_{i}^{2}}+2 \sum_{h>i \in \mathrm{S}_{k}} \frac{t_{k i} t_{k h}}{\pi_{i} \pi_{h}}-2 \frac{N}{N-1} \sum_{h>i \in \mathrm{S}_{k}} \frac{t_{k i} t_{k h}}{\pi_{i h}}\right)$

Under TSS, (7) and (8) are proven to be conservative estimators of the actual variances. The conservative nature of (7) and (8) stems from TSS, owing to the independence among first-phase points (Wolter 1985). Nothing can be said about the properties of (5) and (6) under SGS. In this case the estimation of variance required more refined procedures (Opsomer et al. 2007, Fewster 2011).

Most of NFIs involve two phases of sampling. Relevant examples are the NFIs of Canada (Gillis 2001), USA (Scott et al. 2004), the former Soviet Union (Gabler \& Schadauer 2007), New Zealand (Stephens et al. 2012), Romania and Switzerland (Tomppo et al. 2010). In the recent Italian NFI, three phases of sampling are adopted. In the Italian case, the second-phase points are visited to record the forest type in order to estimates extents, while the totals of forest attributes are estimated from a third-phase sample. The expressions of the third-phase estimators are obviously more cumbersome than those achieved in two phases. Details on thirdphase estimators are given by Fattorini et al. (2006).

\section{The use of auxiliary information}

As already pointed out, NFIs usually require estimates of extents and totals for several forest types, for several regions defined by political subdivisions, for other domains such as ownership categories and silvicultural types and for combinations of them. Practically speaking, thousands of estimates are produced as the output of a NFI. In this framework, statisticians have neither time nor resources to select ad hoc estimators for each survey variable. Therefore the only feasible way is to adopt linear estimators as those in equations (5) and (6), in which the weights attached to sample observations are constructed and applied to all variables and domains. Because these weights are derived from the sampling design, they do not effectively take advantage of the increasing availability of various inexpensive auxiliary data from remote sensing sources (e.g., photo-interpreted land cover class, location, elevation, slope and a sequence of thematic mapping spectral bands).

On the other hand, Opsomer et al. (2007) emphasize the great opportunity to improve the accuracy of NFI estimates making use of auxiliary data derived from remote sensing sources That can be done by calibration procedures in which the original weights are adjusted, making them sum to totals of the auxiliary variables. Opsomer et al. (2007) list a large number of techniques for adjusting sample weights. Most of them make use of super-population models understanding the relationships between key forestry variables and remotely sensed information. All of them adopt these models in the framework of model-assisted estimation, i.e., models are only used to determine the sample weights while the statistical properties of the resulting estimators are derived in a design-based framework from the scheme actually adopted to select the sample points. An application to Northern Utah Mountains data shows estimated efficiencies of the model-assisted estimators which vary from 1.3 to 2 with respect to the traditional FIA estimators (Opsomer et al. 2007).

\section{Estimation of non-forest resources}

During the FAO Expert Consultation on Global Forest Resources Assessment 2000 (Kotka, Finland 1996), the importance of trees outside forests (TOF) and the need for complete and detailed information about these stands were underlined for the first time. NFIs are currently requested to broaden their scopes to include the assessment of TOF attributes (Kleinn 2000, 2002). TOF include small woodlots, three rows, urban forests and isolated trees and play a basic role in biodiversity conservation and carbon sequestration. The main objective of TOF inventories is the estimation of totals and/or averages of some physical attribute of the units (e.g., woodlot size and tree-row length). Probably, an efficient solution would require the use of ad hoc sampling schemes for each of the target parameters. However, in order to save time and resources, it may be appealing to perform the estimation in the first-phase of NFIs, because most physical attributes can be recorded from the aerial information col- lected during the first inventory phase without any field work.

Quoting from Baffetta et al. (2011b), let W be the population of $M$ woodlots, or trees rows or urban forests in the study area. Let $w_{l}, \ldots, w_{M}$ be the sizes of the $M$ units and let $y_{\mathrm{i}}$ be the value of a physical attribute of the $j$ th unit which can be recorded from aerial imagery. Suppose that the population total (eqn. 9):

$$
T_{W}=\sum_{j \in \mathrm{W}} y_{j}
$$

and/or the population mean $\bar{Y}_{W}=T_{W} / M$ are the parameters to be estimated. To this purpose, denote by $\mathrm{G}$ the set of distinct woodlots, tree rows or urban forests which contain at least one of the $N$ first-phase points and let $m$ be the (random) size of G. As proven by Baffetta et al. (2011b), under TSS the quantity (eqn. 10):

$$
\hat{T}_{(1) W}=\frac{R}{N} \sum_{j \in \mathrm{G}} \frac{y_{j}}{w_{j}}
$$

turns out to be an approximately unbiased estimators of $T_{W}$. It is worth noting that (10) avoids the troublesome quantification of the portion of the selected units lying in adjacent quadrats, as would be requested by the genuine Horvitz-Thompson estimator. Moreover (eqn. 11):

$$
V_{(1) W}=\frac{1}{N(N-1)}\left[R^{2} \sum_{j \in \mathrm{G}}\left(\frac{y_{j}}{w_{j}}\right)^{2}-N \hat{T}_{(1) W}^{2}\right]
$$

is proven to be a conservative estimator for the variance of $\hat{T}_{(I) W}$. For $y_{j}$ invariably equal to $1, T_{W}$ coincides with the population abundance $M$ and (10) provides an abundance estimator, say $\hat{M}_{(l)}$. Thus a very natural estimator of $\bar{Y}_{W}=T_{W} / M$ is given by the ratio (eqn. 12):

$$
\hat{\bar{Y}}_{(1) W}=\frac{\hat{T}_{(1) W}}{\hat{M}_{(1)}}
$$

which is approximately unbiased with variance estimator (eqn. 13):

$$
V_{(1) \bar{Y}}=\frac{R^{2}}{\hat{M}_{(1)}^{2} N(N-1)} \sum_{j \in \mathrm{G}}\left(\frac{y_{j}-\hat{\bar{Y}}_{(1) W}}{w_{j}}\right)^{2}
$$

The validity of these estimators is empirically checked by a simulation study (Baffetta et al. 2011b). They are applied to estimate the average size and the abundance of the Italian urban forests from the sample of the 430 urban forests selected throughout Italy by the first-phase points of the last Italian NFI (Corona et al. 2012a).

Regarding isolated trees, their abundance can be estimated from the aerial information acquired during NFIs, even if a further aerial sampling phase is necessary in this case. Baffetta et al. (2011a) propose the use of a second-phase in which the $N$ first-phase points are partitioned into strata by using 
aerial imagery. Usually the strata coincide with land cover classes easily identifiable from the imagery. Then, a second-phase sample of points is selected from each strata by simple random sampling without replacement, a circle of fixed size is centered at the second-phase points and the number of isolated trees within the circle is counted once again from the aerial imagery. When the presence of isolated trees is more likely in some strata, these strata should be more intensively sampled. Moreover, because isolated trees are rare and widely scattered over territories, a suitable choice should be circles of about 100-200 m radius which are much larger than those usually adopted when surveying within forests (10-15 m radius). If $\mathrm{W}$ now denotes the population of $M$ isolated trees over the study area, if $\mathrm{P}_{l}, \ldots, \mathrm{P}_{L}$ denote the $L$ strata in which the population $\mathrm{P}$ of the $N$ first-phase points is partitioned, $N_{l}, \ldots, N_{L}$ denote the stratum sizes, $\mathrm{S}_{l}, \ldots, \mathrm{S}_{L}$ denote the samples of points selected from each stratum and $n_{1}, \ldots, n_{L}$ the sample sizes, the two-phase aerial estimator of $M$ turns out to be (Baffetta et al. 2011a - eqn. 14)

$$
\hat{M}_{(2)}=\frac{R}{b} \sum_{l=1}^{L} p_{l} \bar{m}_{l}
$$

where $b$ is the size of circles, $p_{l}=N_{l} / N, m_{i}$ denotes the number of isolated trees aerially counted within the plot $i$ and $\bar{m}_{l}$ is the average of the $m_{i} \mathrm{~s}$ for $i \in \mathrm{S}_{l}$. The estimator (10) is unbiased with variance which can be unbiasedly estimated by (eqn. 15):

$$
\begin{gathered}
V_{(1) M}=\frac{R^{2}}{b^{2}(N-1)} . \\
{\left[\sum_{l=1}^{L} p_{l}\left(N_{l}-1\right) \frac{s_{l}^{2}}{n_{l}}+\sum_{l=1}^{L} p_{l}\left(\bar{m}_{l}-\hat{M}_{(2)}\right)^{2}\right]}
\end{gathered}
$$

where $s_{l}^{2}$ is the sample variance of the $m_{i}$ s for $i \in \mathrm{S}_{l}$.

Obviously, if totals or averages of some biophysical attributes such as tree volume and biomass are of interest (rather than size or length), subsequent sampling phases must be performed on the field. Corona \& Fattorini (2006) propose the use of two-phase cluster sampling to survey tree rows, while Corona et al. $(2011 \mathrm{~b})$ propose the use of sector sampling to survey woodlots. A third stratified sampling phase is suggested by Baffetta et al. (2011a) for field surveys of isolated trees.

\section{Non-response treatment}

Non-response is often a problem in NFIs. Non-response occurs for two main causes: (a) plots selected in the second phase are located in difficult terrains and cannot be reached by survey crews or, even if reached, the steep slope of the terrain does not allow the recording activities within; (b) plots selected in the second phase are inaccessible because of cultural prohibitions or because landowners deny field crews access. The reasons for non-response are not necessarily the same all over the world. In the USA, (a) is the primary reason for non-response. Procedures for treating non-response due to (a) are proposed by McRoberts (2003) and are not treated here. This section deals with the problem of non-response adjustment when difficult terrains preclude access to plots or recording activities. This is especially relevant in those countries where forest areas are mainly located in mountainous and/or remote areas.

In this framework, non-response problems manly concern the estimation of totals, which involves the recording of forest attributes within the plots centered at second-phase points. On the other hand, as to the estimation of extents, in most situations the forest type at plot centers can be determined some distance away from the points or by means of high resolution aerial image, while the district in which plot centers fall can be even determined from the map.

A vast literature deals with the problem of non-response adjustment by means of several techniques, but most of them are judged unfeasible by Fattorini et al. (2013) for environmental and forest surveys. Widely applied methods to account for unit non-response were recently referred to as non-response propensity weighting by Haziza et al. (2010). These methods view the respondent set as the result of a further phase of sampling, assuming the existence of a response mechanism for which every sampled unit has its own invariably positive response probability. It is also (tacitly) assumed that each unit responds independently to the others. Practically speaking, the respondent set is realized as a sub-sample of the selected sample. A realistic model is adopted to link the unknown response probabilities with some auxiliary variables. The model is subsequently used to estimate the probabilities on the basis of auxiliary information available for all the sampled unit. Unfortunately, in forest inventories responses cannot be viewed as outcomes of dichotomous and independent experiments with unknown probabilities. If some second-phase points cannot be reached, no random experiment can be claimed because they will never be reached. In these situations responses should be viewed as fixed characteristics of the points, in such a way that the population of first-phase points is partitioned into respondent and non-respondent strata. Moreover, the assumption that responses are independent events is likely to be unrealistic in forest surveys. Neighboring points, lying in terrains with the same characteristics, tend to have a similar response pattern, i.e., a sort of spatial contagion is likely to be present among responses. On the basis of these considerations, Fattorini et al. (2013) conclude that the use of non-response propensity weighting in forest inventory does not seems to be logically defensible.

Alternatively, unit non-response could be handled by a plethora of imputation techniques. Generally speaking, imputation is a procedure in which non response values are replaced by substitutes and estimation is performed on the completed data. As pointed out by Särndal \& Lundström (2005), imputed values are artificial and are customarily obtained by means of a prediction model presuming a relationship between the survey variable and a set of covariates known for all the sampled units. In accordance with the presumed model, commonly used techniques of imputation are, e.g., regression imputation, nearest neighbor imputation, hot deck imputation and multiple imputation (for a review see Little \& Rubin 2002). Without entering on these techniques, it should be pointed out that no prediction model can be validated in the set of non-respondent units. On the basis of these consideration Fattorini et al. (2013) conclude that it is difficult to scientifically defend any proposed method/model of imputation.

Because both response and prediction modeling are not sufficiently convincing for non-response treatment in forest inventories, Fattorini et al. (2013) propose the use of a technique recently referred to as non-response calibration weighting (Haziza et al. 2010). Non-response calibration weighting (henceforth NCW) modifies the weights originally attached to each respondent observation. The modified weights are able to estimate without errors the means or totals of a set of auxiliary variables known for all the population units. The rationale behind NCW is obvious: if the calibrated weights guess the means or totals of the auxiliary variables without errors, they should be suitable for estimating the mean or the total of the survey variable, providing a relationship exists between the survey and the auxiliary variables. Noteworthy, the NCW approach does not need to refer explicitly to any model, allowing for a complete design-based treatment in which forest attributes and non-response are both viewed as fixed characteristics.

The theoretical and empirical design-based findings achieved by Fattorini et al. (2013) look promising. NCW accomplishes the goal of reducing non-response bias when the relationships between interest and auxiliary variables are similar in respondent and non-respondent strata. NCW can even increase the accuracy of estimation with respect to the complete-sample estimation when a close linear relationship exists between the survey and auxiliary variables.

As to the choice of auxiliary information to be used in NCW, it should be mainly guided by cautionary, practical considerations about the nature of the auxiliary variables and their relationships with the survey variable. In 
presence of non-response, the crucial point is to eliminate non-response bias, because "if an estimator is greatly biased, it is poor consolation that its variance is low" (Särndal \& Lundström 2005). Thus, the key requiremen is not the strength of the relationships between the survey and auxiliary variables (which affects the sampling variance), but rather the fact that these relationships (linear or not, strong or not) are similar in respondent and non-respondent strata.

Noteworthy, in recent years laser scanning (and, namely, airborne laser scanning, henceforth referred to as ALS) is increasingly being applied to support forest inventories, providing assessment of the height of upper canopy for the surveyed area. A close relationship has been proven between the timber volume (or standing biomass) in inventory plots and the canopy height from ALS surveys (Næsset 2002, 2004, Næsset et al. 2004, Parker \& Evans 2004, Corona \& Fattorini 2008, Gregoire et al. 2011, Corona et al. 2012b). Obviously, this relationship should hold irrespective of the fact that plots can be reached in the field or not. As such, it is likely to be the same in respondent and non respondent stratum. Therefore, the exploitation of canopy height model (CHM) as auxiliary variable in the $\mathrm{NCW}$ approach seems to be a promising strategy to account for non-response in NFIs. It has been recently investigated by Corona et al. (2014). Because the population of first-phase points is random - being the outcome of the first sampling phase - additional methodological refinements are necessary to apply NCW in forest inventories with respect to the standard methodology proposed by Fattorini et al. (2013) in which samples are selected from a fixed population.

Quoting from Corona et al. (2014), denote by $\mathrm{R}$ the set of second-phase points reached in the field out of the $n$ points of the secondphase sample $\mathrm{S}$ and denote by $\mathbf{x}_{i}=\left[1, h_{i}\right]^{\mathrm{T}}$ the vector where $h_{i}$ denotes the sum of CHM values for all the pixels belonging to the inventory plot centered at the $i$-th point. If the $\mathbf{x}_{i}$ S are available for each point $i \in \mathrm{P}_{F}$, the mean vectors (eqn. 16)

$$
\overline{\mathbf{X}}_{k}=\frac{1}{N_{F, k}} \cdot \sum_{i \in \mathrm{P}_{F, k}} \mathbf{x}_{i}=\left[1, \bar{H}_{k}\right]^{T}
$$

are known for each $k=1, \ldots, K$, where $\mathrm{P}_{F, k}$ is the set of the $N_{F, k}$ first-phase points lying in the $k$-th forest type/district, and $\bar{H}_{k}$ is the mean of the CHM values within the plots centred at these points. Then $\overline{\mathbf{X}}_{k}$ can be used in the calibration estimator of $T_{k}$, say (eqn. 17):

$$
\hat{T}_{(2) C A L, k}=\frac{R}{a} p_{F, k} \hat{\mathbf{b}}_{R, k}^{\mathrm{T}} \overline{\mathbf{X}}_{k}
$$

where $p_{F, k}=N_{F, k} / N$ and (eqn. 18):

$$
\hat{\mathbf{b}}_{R, k}=\left(\sum_{i \in \mathrm{R}_{k}} \frac{\mathbf{x}_{i} \mathbf{x}_{i}^{\mathbf{T}}}{\pi_{i}}\right)^{-1} \sum_{i \in \mathrm{R}_{k}} \frac{t_{i k} \mathbf{x}_{i}}{\pi_{i}}
$$

and $\mathrm{R}_{k} \subset \mathrm{R}$ denotes the sub-sample of second-phase points reached in the field among the second-phase points lying in the $k$-th forest type/district. If the relationship of the forest attribute with CHM height is similar for the respondent and non-respondent points of $\mathrm{P}_{F, k}$, then $\hat{T}_{(2) C A L, k}$ constitutes an approximately unbiased estimator of $T_{k}$. The authors also propose three conservative estimator of the variance of $\hat{T}_{(2) C A L, k}$.

The simulation of a two-phase forest inventory performed by Corona et al. (2014) gave positive insights on the effectiveness of the calibration estimator in reducing the bias induced by non-response, also providing performance comparable with or even better than that achieved by the complete sample estimator. A set of non-response zones were scattered over the forest area, giving rise to a percentage of non-response points of about $9 \%$. The relative downward bias due to nonresponse was of about $13 \%$, which reduced to $0.2 \%$ by the use of calibration estimator with a relative error of $2.2 \%$. Interestingly the calibration estimator showed a performance comparable with that achieved in the case of a complete response. In that case the bias was absent with a relative error of $2.9 \%$. The sole shortcoming of the procedure was the estimation of variance and the subsequent construction of confidence intervals. Indeed, the proposed variance estimators largely overestimated the actual variance, in such a way that the actual gain achieved by the calibration procedure remained undetected by the variance estimates. The construction of less biased variance estimators seems to be one of the necessary steps toward a suitable implementation of this novel idea.

\section{Conclusions}

NFIs based on two-phase sampling strategies with first-phase points selected by means of stratified or systematic schemes and second-phase points selected ignoring non-forest points ensure a statistically sound estimation of extents and totals for all variables and domains. From a design-based point of view, the resulting estimators are indeed approximately unbiased and conservative estimators of their variances are available. Moreover, information achieved in the first inventory phase can be used to estimate totals and averages of physical attributes of TOF without any additional field work. Inexpensive auxiliary data from remote sensing and aerial sources can be used at estimation level, i.e., without any modification of the inventory field protocols, as auxiliary information to improve the accuracy of the estimates. Among these information, canopy height from ASL data seems to be the most promising, being able to improve accuracy and, at the same time, to reduce the bias due to missing observations occurred at those points that cannot be reached by forest crews.

Even if canopy height is often available at low or even no cost from ALS surveys carried out on large territories for purposes other than forestry applications, e.g., topographical or hydrogeological surveys (Montaghi et al. 2013), the availability of ASL data for a whole country (as should be necessary for applications in NFIs) rarely occurs at the moment. However, some countries have already completed national wall-to-wall ASL surveys over their territories, e.g., The Netherlands (Swart 2010), Sweden (Petersen \& Rost 2011), Finland (http://www.maanmittauslaitos.fi/en/maps-5), Denmark (http:// www.sharpgis.net/post/2008/10/24/First-cou ntry-to-be-fully-mapped-in-3D.aspx), Switzerland (http://www.swisstopo.admin.ch). If this tendency will be confirmed in the future, ASL data are destined to play a basic role in NFI estimation.

\section{Acknowledgments}

The author thanks Gherardo Chirici from the University of Molise, Isernia, Italy, and Piermaria Corona from Consiglio per la Ricerca e Sperimentazione in Agricoltura (CRA), Arezzo, Italy for their support and suggestions on issues regarding forest surveys and Luca Pratelli from Naval Academy, Livorno, Italy, for his continuous support on the theoretical issues.

\section{References}

Baffetta F, Corona P, Fattorini L (2011a). Assessing the attributes of scattered trees outside the forest by a multi-phase sampling strategy. Forestry 84: 315-325. - doi: 10.1093/forestry/cpr015 Baffetta F, Fattorini L, Corona P (2011b). Estimation of small woodlot and tree row attributes in large scale forest inventories. Environental and Ecological Statistics 18: 147-167. - doi: 10.10 07/s10651-009-0125-0

Barabesi L (2003). A Monte Carlo integration approach to Horvitz-Thompson estimation in replicated environmental designs. Metron 61: $355-$ 374.

Barabesi L, Marcheselli M (2003). A modified Monte-Carlo integration. International Mathematical Journal 3: 555-565.

Barabesi L, Franceschi S (2011). Sampling properties of spatial total estimators under tessellation stratified designs. Environmetrics 22: 271 278. - doi: 10.1002/env.1046

Corona P (2000). Introduzione al rilevamento campionario delle risorse forestali [Introduction to sampling of forest resources]. Edizioni CUSL, Firenze, Italy, pp. 284. [in Italian].

Corona P, Fattorini L (2006). The assessment of tree row attributes by stratified two-stage sampling. European Journal of Forest Research 125: 
57-66. - doi: 10.1007/s10342-005-0078-2

Corona P, Fattorini L (2008). Area-based LiDARassisted estimation of forest standing volume. Canadian Journal of Forest Research 38: 29112916. - doi: 10.1139/X08-122

Corona P, Marchetti M (2007). Outlining multipurpose forest inventories to assess the ecosys tem approach in forestry. Plant Biosystems 141: 243-251. - doi: 10.1080/11263500701401836

Corona P, Fattorini L, Franceschi S (2011a). Twostage sector sampling for estimating small woodlot attributes. Canadian. Journal of Forest Research 41: 1819-1826. - doi: 10.1139/x11-101

Corona P, Chirici G, McRoberts RE, Winter S, Barbati A (2011b). Contribution of large-scale forest inventories to biodiversity assessment and monitoring. Forest Ecology and Management 262: 2061-2069. - doi: 10.1016/j.foreco.2011.08. 044

Corona P, Agrimi M, Baffetta F, Barbati A, Chiriacò MV, Fattorini L, Pompei E, Valentini R, Mattioli W (2012a). Extending large-scale forest inventories to assess urban forests. Environmental Monitoring and Assessment 184: 1409-1422. - doi: 10.1007/s10661-011-2050-6

Corona P, Cartisano R, Salvati R, Chirici G, Floris A, Di Martino P, Marchetti M, Scrinzi G, Clementel F, Travaglini D, Torresan C (2012b) Airborne laser scanning to support forest resource management under alpine, temperate and $\mathrm{Me}$ diterranean environments in Italy. European Journal of Remote Sensing 45: 27-37. - doi 10.5721/EuJRS20124503

Corona P, Chirici G, Franceschi S, Maffei D, Marcheselli M, Pisani C, Fattorini L (2014). Design-based treatment of missing data in twophase forest inventories using canopy height from laser scanning. Canadian Journal of Forest Research (early view). - doi: 10.1139/cjfr-20130521

de Vries PG (1986). Sampling theory for forest inventory. Springer, Berlin, Germany, pp. 399.

Fattorini L, Marcheselli M, Pisani C (2004). Twophase estimation of coverages with second-phase corrections. Environmetrics 15: 357-368. - doi: 10.1002/env.647

Fattorini L, Marcheselli M, Pisani C (2006). A three-phase sampling strategy for large-scale multiresource forest inventories. Journal of Agricoltural Biological and Environmental Statistics 11: 1-21. - doi: 10.1198/108571106X96871

Fattorini L, Franceschi S, Maffei D (2013). Design-based treatment of unit nonresponse in environmental surveys using calibration weighting. Biometrical Journal 55: 925-943. - doi 10.1002/bimj.201100262

Fewster RM (2011). Variance estimation for systematic designs in spatial surveys. Biometrics 67: 1518-1531. - doi: 10.1111/j.1541-0420.2011 .01604.x

Gabler K, Schadauer K (2007). Ansätse und Stichprobenpläne nationale Forstinventuren [So- me approaches and designs of sample-based national forest inventories]. Austrian Journal of Forest Science 124: 105-133. [in German].

Gillis MD (2001). Canada's national forest inventory (responding to current information needs). Environmental Monitoring and Assessment 67: 121-129. - doi: 10.1023/A:1006405820244

Gregoire TG, Valentine HT (2008). Sampling strategies for natural resources and the environment. Chapmam \& Hall, Boca Raton, FL, USA, pp. 492 .

Gregoire TG, Ståhl G, Næsset E, Gobakken T, Nelson R, Holm S (2011). Model-assisted estimation of biomass in a LiDAR sample survey in Hedmark County, Norway. Canadian Journal of Forest Research 41: 83-95. - doi: 10.1139/X10195

Haziza D, Thompson KJ, Yung W (2010). The effect of nonresponse adjustments on variance estimation. Survey Methodology 36: 35-43. [online] URL:

http://www.publications.gc.ca/collections/collection_2010/statcan/12-001-X/12-001-x2010001eng.pdf

Kleinn C (2000). On large area inventory and assessment of trees outside forests. Unasylva 51: 310.

Kleinn C (2002). New technologies and methodologies for national forest inventories. Unasylva 53: 10-15.

Little RJA, Rubin DB (2002). Statistical analysis with missing data ( $2^{\text {nd }}$ edn). Wiley, New York, USA, pp. 381.

Maffei D (2011). Design-based treatment of nonresponse in large-scale forest inventories: an application to the Italian National Forest Inventory. $\mathrm{PhD}$ thesis, Department of Statistics, University of Florence, Florence, Italy, pp. 79.

Mandallaz D (2008). Sampling techniques for forest inventories. Chapman \& Hall, Boca Raton, FL, USA, pp. 256.

McRoberts RE (2003). Compensating for missing plot observations in forest inventory estimation. Canadian Journal of Forest Research 33: 19901997. - doi: 10.1139/x03-112

Montaghi A, Corona P, Dalponte M, Gianelle D, Chirici G, Olsson H (2013). Airborne laser scanning of forest resources: an overview of research in Italy as a commentary case study. International Journal of Applied Earth Observation and Geoinformation 23: 288-300. - doi: 10.1016/j.jag.2012.10.002

Næsset E (2002). Predicting forest stand characteristics with airborne scanning laser using a practical two-stage procedure and field data. Remote Sensing of Environment 80: 88-99. - doi: 10.1016/S0034-4257(01)00290-5

Næsset E (2004). Practical large-scale forest stand inventory using small-footprint airborne scanning laser. Scandinavian Journal of Forest Research 19: 164-179. - doi: 10.1080/0282758031 0019257
Næsset E, Gobakken T, Holmgren J, Hyyppä H, Hyyppä J, Maltamo M, Olsson H, Persson A, Söderman U (2004). Laser scanning of forest resources: the Nordic experience. Scandinavian Journal of Forest Research 19: 482-499. - doi: 10.1080/02827580410019553

Opsomer JD, Breidt FG, Moisen GG, Kauermann G (2007). Model-assisted estimation of forest resources with generalized additive models. Journal of the American Statistical Association 102: 400-416. - doi: 10.1198/016214506000001491

Parker RC, Evans DL (2004). An application of LiDAR in a double-sample forest inventory. Western Journal of Applied Forestry 19: 95-101. Petersen YM, Rost HB (2011). Swedish lidar project. New nationwide elevation model. GIM International 25, web site. [online] URL: http:// www.gim-international.com/issues/articles/id16 64-Swedish_Lidar_Project.html

Särndal CE, Lundström S (2005). Estimation in survey with nonresponse. Wiley, New York, USA, pp. 199.

Särndal CE, Swensson B, Wretman J (1992). Model-assisted survey sampling. Springer-Verlag, New York, USA, pp. 694.

Schreuder HT, Gregoire TG, Wood GB (1993). Sampling methods for multiresource forest inventory. Wiley, New York, USA, pp. 446.

Scott CT, Bechtold WA, Reams GA, Smith WD, Hansen MH, Moisen GG (2004). Sample-based estimators utilized by the forest inventory and analysis national information management system. In: "The Enhanced Forest Inventory and Analysis Programmational Sampling Design and Estimation Procedures" (Bechtold WA, Patterson PL eds). Southern Research Station, USDA Forest Service, Asheville, NC, USA, pp. 43-68. Stephens PR, Kimberley MO, Beets PN, Paul TSH, Searles N, Bell A, Brack C, Broadley J (2012). Airbone scanning LiDAR in a double sampling forest carbon inventory. Remote Sensing of Environment 117: 348-357. - doi: 10.10 16/j.rse.2011.10.009

Swart LMT (2010) How the up-to-date height model of the Netherlands (AHN) became a massive point data cloud. In: "Management of massive point cloud data: wet and dry" (van Oosterom PJM, Vosselman MG, van Dijk TAGP, Uitentuis $\mathrm{M}$ eds). Netherlands Geodetic Commission, Delft, The Netherlands, pp. 17-32. [online] URL: http://www.swartvast.nl/rapporten /AHN_as_massive_point_cloud_data_LMTh_S wart.pdf

Tomppo E, Gschwantner LM, McRoberts R (2010). National forest inventories: pathways for common reporting. Springer, Heidelberg, Germany, pp. 612.

Wolter KM (1985). Introduction to variance estimation. Springer-Verlag, New York, USA, pp. 427. 